\title{
S45. Psychogeriatrics
}

DEVELOPMENT AND AGING OF THE COCHLEAR NUCLEUS. QUALITATIVE AND QUANTTTATIVE IMMUNOHISTOCHEMICAL STUDY.

\section{JALENOUES,MD.Ph.D;AJ.COUDERT,M.D,R.ROMAND,Ph.D. Servioe dePsychiarie, OHUde Cemmont-Ferandet Laboratire de Nenobiobgie, UFR des Céarux, Aubìze, France.}

We realized an immunohistochemical study of the localization of the glial fibrillary acidic protein (GFAP) in cochlear nucleus (CN) during development and aging in rats.

We used various immunohistochemical methods: vibratome, cryostat sections on a freezing microtome, and paraffin with different fixation procedures. We studied various stages of development (days 1, 5, 10 and 21), adult stage and aging. We realized a qualitative and quantitative study of $\mathrm{CN}$ astrocytes and neurons in developing, adult and old rats.

We note the same kinds of type II multipolar astrocytes on paraffin sections as on cryostat sections. The distribution of the immunoreactive cells varies according to stages and area. During the postnatal period, GFAP immunoreactivity is discrete and preferentially distributed in periphery of $\mathrm{CN}$. It increases until postnatal day 21. In adult rat, its distribution in $\mathrm{CN}$ is heterogeneous. In DCN, GFAP immunoreactivity decreases from molecular to polymorphic layer and from dorsal to ventral area. In PVCN, its density is lower and decreases along a dorso-ventral axis. GFAP immunoreactivity density is maximum in AVCN where it decreases along the dorso-ventral and rostro-caudal axes. Granule cells domain shows a high density of astrocytes. During aging, the density and fibrous aspect of immunoreactive astrocytes is highly increased. Their distribution in $\mathrm{CN}$ is homogeneous. The density of neurons decreases in AVCN of old rats.

These first results show and compare the distribution of astrocytes in $\mathrm{CN}$ of developing, adult and old rats. In old rats, the decrease of number of neurons in AVCN, compared to neuronal stability in other regions, is very interesting data. This is a way of comparing astrocytic reaction in two different regions of $\mathrm{CN}$ in old rats. But the impact of peripheral presbycusis must be taken into account before interpreting results.
SYSTEMIC CHANGES OF PARAMETERS OF THE GLUCOSE METABOLISM IN PATIENTS WITH DEMENTIA OF ALZHEIMER'S TYPE (DAT)

I Thome, L Frölich, Y Taneli, GA Wiesbeck, P Riederer

Departement of Psychiatry (Chair: Prof. Dr. H. Beckmann), University of Würzburg, Füchsleinstraße 15, D-97080 Würzburg, Germany

Changes of cerebral glucose metabolism in DAT have been documented both in vivo and in post mortem brain tissue. Recently, evidence has been provided for a derangement of glucose metabolism outside the CNS in the peripheral blood, i.e. both hypoglycemia and hyperinsulinemia. At present, the hypothesis of a general systemic defect of cell metabolism involving glucose metabolism and insulin receptor function in DAT is under debate.

In the present study in 16 patients with DAT and 16 non-demented, healthy, age-matched controls we have performed an oral glucose tolerance test (OGTT). After overnight fasting, a 100g OGTT was conducted. Blood was collected before and 30,60,90,120,180 and $240 \mathrm{~min}$ after the glucose challenge. Plasma glucose, insulin, glucagon and C-peptid were determined. In the fasting plasma, levels of $\mathrm{HbAlc}$ were also measured.

The results indicate that indices of a regulatory disturbance of glucose metabolism can be found in the peripheral blood in DAT. This suggests that the known changes of cerebral glucose and energy metabolism could reflect a primary cellular abnormality relevant for the pathogenesis of DAT. If these changes also involve a defect of the insulin receptor function in DAT need to be demonstrated by further studies. Furthermore, the specifity of these changes has to be elucidated. 
SLEEP RESPONSE TO A SINGLE-DOSE OF ANTIDEPRESSANT IN DEPRESSIVE PSEUDO-DEMENTIA

D. Pringuey, C. Capdeville, J. L. Belugou, L. Talichet and G. Darcourt. Department of Psychiatry. C.H.U. Pasteur.

BP 69 - 06002 - Nice cedex 1 - France

In depressive pseudo-dementia (1) the distinction between depression and dementia can be stressed by sleep studies on the basis of the occurence of a paradoxical enhancement of REM sleep in depression opposed to the observation of a progressive loss of REM sleep in dementia (2). In order to clarify borderline sleep-EEG profile (3), we studied the sleep pattern response to antidepressant drugs and previously demonstrated in depressed patients that an evening dose of trimipramine, a tricyclic sedative antidepressant, may provide additional information on baseline data (4)

Severely impaired inpatients over 60 years old, meeting both DSM III R criteria of depression and dementia completed clinical evaluation (MMS, Hamilton depression scale), laboratory tests (biochemistry screening, thyroid function), CT scan and a withdrawal of medications of at least 15 days. EEG sleep were recorded on a 4 channel portable system (Medilog recorder), over two consecutive nights : one base-line night and one single-dose test night after an evening dose of Trimipramine (50 mg) Sleep records were visually scored according to standard methods. The response pattern to the drug was taken into account to conduct a new antidepressant treatment strategy or a prolonged withdrawal of drugs.

On 11 consecutively admitted patients, significant changes in sleep parameters under the drug test, mainly related to a SWS restauration, announced a later clinical recovery on a new active treatment in 6 patients. Responders exhibited a sedative effect, a mild increase in sleep efficacy, an increase in number of stage changes, a clear Rem suppression and an acute SWS restauration (baseline SWS time $=4 \pm 2.6 \mathrm{vs}$ trimi $=12 \pm 7,2$ p .005 Wilcoxon test; base line SWS $\%=4.09 \pm 2.55$ vs trimi $=$ $12.05 \pm 7.4$ p<.05).

A pharmacological sensitization of sleep studies using antidepressant as a probe may improve the diagnostic discrimination between dementia and depression.

1-Reynolds C.F. et al (1988) Arch. Gen. Psychiatry 45, 258-264

2-Benca R.M. et al (1992) Arch. Gen. Psychiatry 49 : 651-668.

3- Bliwise D.L. (1993) Sleep 16,40-81.

4 - Feuillade Ph, et al (1992) J. Aff. Dis. 24, 135-146.

\section{OVERLAP AND DIFFERENCES IN GERIATRIC DEPRESSION AND SENILE DEMENTIA OF ALZHEIMER-TYPE: A FAMILY STUDY PERSPECTIVE}

RHeun. M. Burkart, W. Maier

Department of Psychiatry, University of Mainz

Untere Zahlbacher Straße 8, D-6500 Mainz, Germany

Senile dementia of Alzheimer-type and geriatric depression show a large amount of symptomatic overlap which might be due to simple symptomatic similarities in two distinct disorders, but might also bc the consequence of an enhanced comorbidity of two distinct disorders caused by common familial factors. To evaluate the role of familial factors, the study compares the familial loads of psychiatric disorders and of cognitive deficits in both patient groups and in healthy controls. - Patients were recruited, if they fulfilled the following DSM-III-R-criteria: diagnosis of depression (age at onset $>=60 y$ ) or senile dementia of Alzheimer-type. A control group was recruited out of the general population. Probands and first-degree relatives were examined with the Composite International Diagnostic Interview (CIDI). Relatives older than 55 years were additionally examined for cognitive deficits using several neuropsychologic tests. - The present evidence suggests that dementia and geriatric depression can be distinguished by their familial loads for depression and by the frequencies of minor cognitive deficits, however both disorders show some overlap in their familial loads for dementia.

\section{COMPARISON OF THE EFFICACY OF MOCLOBEMIDE AND FLUVOXAMINE IN ELDERLY PATIENTS WITH A SEVERE DEPRESSIVE EPISODE}

\author{
J.-Ph. Bocksberger ${ }^{*}$ J.-P. Gachoud ${ }^{* *}$,J. Richard*,$P$ \\ Dick $^{*}$ \\ * Clinique Universitaire de Psychiatrie Gériatrique, \\ I.U.P.G., 1225 Geneva, Switzerland \\ ** Roche Pharma (Schweiz) AG, 4153 Reinach, \\ Switzerland
}

In a double blind study performed with elderly patients the efficacy and tolerability of the new antidepressant moclobemide was compared. Moclobemide belongs to a new class of substances called RIMA (Reversible Inhibitor of the monoamine oxidase type A). Fluvoxamine, a selective reuptake-inhibitor of 5-HT, belongs to a class of antidepressants known for their better tolerability compared to tricyclic especially with elderly patients. 40 elderly patients (mean age 75 years) with major depression (according to DSM-III) were randomized to receive either moclobemide $(300 \mathrm{mg})$ or fluvoxamine $(100 \mathrm{mg})$ twice daily. Dosages were increased when necessary on day 8 , to a maximum of moclobemide $450 \mathrm{mg}$ or fluvoxamine $200 \mathrm{mg}$ and in most cases were maintained at these levels for the remainder of the study period (4 weeks). Moclobemide was more effective than fluvoxamine showing a marked antidepressant effect and an earlier effect on psychomotor retardation. The two drugs were well tolerated showing a low incidence of side effects.

\section{THE USE OF PSYCHOTROPIC DRUGS}

AMONG RESIDENTS IN OLD AGE HOMES

S Weyerer

Central Institute of Mental Health, P.O.Box 122120

D-68072 Mannheim, Germany.

Several community studies indicate that the use of psychotropic drugs increases with advancing age. Compared to that for residents living in private households, the prevalence of psychiatric disorders is significantly higher among residents in homes for the elderly. Therefore, if is of particular interest to study the clinical and social factors that might affect the use of psychotropic drugs among the institutionalized elderly.

In 1988, 542 residents ( 65 and older) in 12 homes for the elderly in the city of Mannheim were interviewed by psychiatrists and clinical psychologists. The head of home recorded for each resident information on their mental state, problem behaviour, Activities of Daily Living (ADL) impairment, and use of psychotropic drugs.

$42.1 \%$ of the home residents had received psychotropic medication (within four weeks prior to interview), showing a wide variation of prescription among the individual homes $(18.2-58.3 \%) .13 .3 \%$ received neuroleptics, $13.0 \%$ tranquilizers, $11.7 \%$ hypnotics and $8.9 \%$ antidepressants. In contrast to results from community studies psychotropic drug use was not associated with sex and mental health problems. Nor was there any significant relationship to age, education, ADL impairment, length of stay and home visits.

From a cross-sectional study we cannot conclude whether the high use of psychotropic drugs is due to institutional conditions or whether it is already high at the time of home admission. Therefore, in a second study we examined a consecutive cohort of residents ( $n=239 ; 65$ and older) at the time of home admission. Among this group the prevalence of psychotropic drug consumption (total: $38.0 \%$; neuroleptics: $13.4 \%$; tranquilizers: $10.2 \%$; hypnotics: $10.2 \%$; antidepressants: $8.6 \%$ ) was very similar to that for the residents examined within the framework of our cross-sectional study. Apparently the use of psychotropic drugs is already high at the time of home admission and unrelated to length of institutionalization.

In order to clarify the temporal relationship between the use of medication and psychiatric disorders a prospective longitudinal design should be applied. Furthermore, there is a need of intervention studies in order to improve the indication of psychotropic drugs among the institutionalized elderly. 
SERUM CONCENTRATIONS OF PERPHENAZINE AND HALOPERIDOL IN THE TREATMENT OF GERONTOPSYCHIATRIC PATIENTS

R Rosenberg. OV Olesen, $S$ Juul Nielsen

Institute for Basic Research in Psychiatry, Department of Biological Psychiatry, Psychiatric Hospital in Aarhus, Skovagervej 2, DK-8240 Risskov, Denmark

Therapeutic drug monitoring has gained increasing acceptance in the treatment with low-dose neuroleptics and tricyclic antidepressants due to very large intraindividual differences in the serum concentrations when patients are treated with standard dosages. By use of TDM, insufficiently low as well as excessively high serum concentrations may be avoided from the beginning of the treatment, i.e. before the appearance of clinical improvement or troublesome side effects.

We have studied serum concentrations in 48 patients (age: 60-95 $\mathrm{yr}$ ), admitted to a gerontopsychiatric department with well-defined catchment area and admission criteria. TDM is used on a regular basis in the drug treatment of the elderly. The indications for neuroleptic treatment were manic symptoms, hallucination or delusion, or behavioral disturbances. Clinically significant improvement was observed in $80 \%$ of the patients. Perphenazine and haloperidol were prescribed at fairly low doses, i.e. mean 7.6 (range 214) $\mathrm{mg} / 24 \mathrm{~h}$ and mean 3.3 (range $1-7.5$ ) $\mathrm{mg} / 24 \mathrm{~h}$, respectively. The mean serum concentration of perphenazine was $2.4 \mathrm{nmol} / 1$ (SD: 2.6 ) and of haloperidol $4.5 \mathrm{nmol} / 1$ (SD: 2.0). Thus, all patients in haloperidol treatment and $52 \%$ of those in perphenazine treatment had serum concentrations below the therapeutic range recommended for treatment of psychotic patients.

The limitations of a naturalistic study should be underlined, but our results strongly indicate that a significant proportion of gerontopsychiatric patients can be appropriately treated with perphenazine and haloperidol at low dosages.

\author{
NEW DRUG DEVELOPMENT - ALZHEIMER'S DISEASE \\ J.T. Apter \\ Princeton Biomedical Research, Princeton, New Jersey, 08540, USA \\ Tacrine (Cognex $($ ) is now FDA approved and is currently the only drug \\ specific for Alzheimer's disease. Because of its frequency and the disability \\ it causes many new drugs are currently in development specifically for this \\ condition. The mechanisms of action of these drugs will be discussed (10 \\ different mechanisms) and 20 different drugs in development will be \\ mentioned. Although none represent a cure, new drug development in \\ Alzheimer's Disease is entering a new era.
}

